\title{
Improving diabetes care in Sri Lankan children: the way forward
}

\author{
Navoda Atapattu ${ }^{1}$, K S H de Silva ${ }^{2}$ \\ Sri Lanka Journal of Diabetes, Endocrinology and Metabolism 2012; 2: 35-38 \\ (Index words: Type 1 diabetes mellitus, children, management)
}

\begin{abstract}
Type 1 diabetes mellitus (T1DM) is an autoimmune disease. The incidence of T1DM is on the increase worldwide. The incidence in Sri Lanka is yet to be determined but there has been a definite increase in its occurrence in the recent past. Uncontrolled diabetes gives rise to micro vascular and macrovascular complications. Various strategies are being implemented to improve care for children with T1DM worldwide. Intensive blood glucose control, regular blood glucose monitoring, screening for complications are prerequisites for better diabetes management. The diabetic care of Sri Lankan children needs to be optimised. Compared to the West we do encounter many practical difficulties. This article discusses the facilities that we have compared to other countries who have achieved good glycaemic control in children and the way forward for the Sri Lankan paediatric population.
\end{abstract}

\section{Introduction}

The incidence of type 1 diabetes mellitus (T1DM) is on the increase especially in the $<5$ year age group (1). Even though there are geographical differences in the trends, the overall annual incidence is estimated to be around 3\%. The incidence of T1DM in Sri Lanka is yet to be determined but over the past few years there has been a definite increase in the number of children affected.

\section{Molecular genetic basis of T1DM}

T1DM is an autoimmune disease which results from destruction of pancreatic beta cells. Eighty five to ninety percent (85\% - 90\%) of patients with T1DM will have islet cell, GAD, IA - 2 , IA - $2 \beta$ or insulin auto antibodies by the time they develop fasting hyperglycaemia (2). The susceptibility to develop T1DM is determined by more than 40 different genomic loci, HLA having the strongest association (3). HLA - DQB1, DQA1 and DRB1 loci are major determinants.

T1DM can be associated with other autoimmune diseases. One third of patients with T1DM who have the HLA genotype HLA-DR3-DQ2/HLA-DR3-DQ2 have transglutaminase autoantibodies (4). The highest risk for the development of both Addison disease and T1DM is associated with a heterozygous HLA-DR4-DQ8/HLA DR3-DQ2 genotype (5). Environmental triggers involved in beta cell destruction are largely unknown except for an association with enteroviral infection.

\section{Neonatal diabetes}

Neonatal diabetes is defined as insulin sensitive hyperglycaemia diagnosed within the first 6 months of life. The incidence is 1 in 400,000 live births. It could be transient or permanent. Permanent neonatal diabetes is found to be associated with mutations in several different genes, namely KCNJ11 gene which encodes ATPsensitive $\mathrm{K}^{+}$channel subunit Kir 6.2 (6), glucokinase (GCK) (7), and transcription factors insulin promoter factor (IPF) (8).

KCNJ 11 mutation reduces the ability for ATP to close the ATP - sensitive $\mathrm{K}^{+}$channel. Consequently, the mutated KATP channels stay open in spite of an increase in intracellular ATP concentration, preventing insulin release in response to high blood glucose levels. It is the commonest cause of permanent neonatal diabetes. Sulfonylureas stimulate insulin secretion by binding to the beta cell's high affinity sulfonylurea receptor and closes the ATP - sensitive $\mathrm{K}^{+}$channels by an ATP independent mechanism. According to recent research it has been suggested that neonatal diabetes diagnosed within 24 weeks after birth who are negative for type 5 diabetes associated antibodies warrant screening for this mutation as these children could be managed effectively with oral medication (6). A proportion of these patients can have associated developmental delay, muscle weakness or epilepsy.

GCK and IPF related neonatal diabetes need lifelong insulin treatment and IPF related patients have both exocrine and endocrine defects. GCK mutation positive

${ }^{1}$ Senior Registrar in Paediatric Endocrinology, Lady Ridgeway Hospital, Colombo, ${ }^{2}$ Consultant Paediatrician, Lady Ridgeway Hospital and Professor in Paediatrics, Faculty of Medicine, University of Colombo, Sri Lanka. 
patients generally have intrauterine growth retardation and glucose intolerant parents.

Permanent neonatal diabetes can occur as part of IPEX syndrome (immunodysregulation, polyendocrinopathy, and enteropathy, X-linked) (9) associated with mutation in FOXP3 or the Wolcott-Rallison Syndrome (10) which is the result of mutation in eukaryotic transcription factor-2 $\alpha$ kinase 3 ( EIF2AK3).

Wolkott-Rallison syndrome has associated epiphyseal dysplasia, osteoporosis and growth retardation occurring at a later age. Other frequent multisystem manifestations include hepatic and renal dysfunction, mental retardation and cardiovascular abnormalities.

Both these conditions are inherited in an autosomal recessive manner and have a poor prognosis. It is important to make the diagnosis in order to counsel families regarding the future and the risk of recurrence.

\section{Insulin therapy and blood glucose monitoring}

Lifelong insulin replacement is needed in T1DM. The Diabetes Control and Complications Trial (DCCT) and its follow up Epidemiology of Diabetes Interventions and Complications (EDIC) study confirmed that intensive glucose control reduce the long term complications and delay the progression of existing complications in T1DM in children $(11,12)$. Continuous subcutaneous insulin infusion (CSII) has become the best insulin delivery system as it simulates the physiological insulin secretion in the body. Other than this basal bolus therapy is widely used as it is much cheaper than CSII but gives the patient a good glycaemic control. In basal bolus regimens and CSII insulin analogues are used. Therapy with insulin analogues is associated with less risk of hypoglycaemia but is expensive. Basal bolus therapy allows flexible meal patterns, insulin administration just after finishing the meal in toddlers, exercise related titration of insulin doses and more effective sick day management. At present the only method of delivery of insulin is via an injection. Although various child friendly devices with small needles, covers to hide needles are available, only the insulin pens are available in Sri Lanka

Methods of blood glucose monitoring have improved and currently continuous glucose monitoring (CGMS) is being used in developed countries to optimise the glucose control.

In addition there are newer glucometers which could be downloaded to a computer. Some glucometers have facilities to calculate correction bolus of insulin at meal time once the meter is programmed for the patient's needs. Some have the facility to check blood ketone levels which is very useful for sick day management.

In Sri Lanka twice daily insulin regime is used which is the cheapest available method. This regime has an increased risk of hypoglycaemia given the profile of action of intermediate acting insulin. In addition it is necessary to give two injections and meals and snacks taken at the same time of the day which would be difficult at times especially for school children. Also it is quite difficult to adjust insulin to cover physical activity.

Ideally at least 4 blood glucose measurements should be done with twice daily or multiple daily insulin therapy optimise the glucose control. In Sri Lanka patients have to bear the cost of the glucometer and the strips unlike in some western countries, which is a major financial burden for most of our parents. Facilities for meticulous blood glucose monitoring must be available for better insulin delivery methods to be instituted. Glycosylated haemoglobin (HbA1c) should be done every 3 months to check the long term diabetes control. Ideally it has to be kept $<7.5 \%$ in all children and adolescents according to ISPAD (International Society of Paediatric and Adolescent Diabetes) Guideline 2009.

Complication screening is done annually after 5 years of diagnosis or after 12 years of age whichever comes earlier. In the West screening is done for celiac disease at diagnosis and every 3 years thereafter. We do the annual screening according to the suggested international guidelines however, the non-availability of HbA1c in the state sector hospitals is a major drawback. We do not routinely screen for celiac disease as it is uncommon in Sri Lanka. However, it could be important that we do screen our children for celiac disease at the diagnosis as the dietary advices need to be given accordingly. Untreated celiac disease not only results in poor glycaemic control but also other long term complications like intestinal malignancies may be over looked.

\section{Nutrition and growth}

Nutrition is an important aspect in children as it is necessary to maintain a normal body mass index (BMI). The paediatric dietician gets involved in the care of these children from the time of diagnosis. In the West after the initial counselling the dietician does a home visit and the patients and their parents are educated on a healthy diet, how to calculate the amount of carbohydrate in different food items and food items that could be used to treat hypoglycaemia. Following this they provide constant evaluation and help for these young patients and their families. Carbohydrate counting is very important in multiple daily injection regimes or for those who are on insulin pumps. It is the trained paediatric dietician who is capable of supporting the children and families with the diet taking in to account the child's weight, eating pattern and activity level. Our parents are advised on a "portion diet” by the paediatrician or the diabetes educator nurse based on body weight and caloric requirement.

\section{Hypoglycaemia}

With newer insulin analogues the risk of hypoglycaemia is less but some patients do experience it. 
This is a very traumatic experience for both the parents and patients. The fear of hypoglycaemia adversely affects diabetes control. Constant advice and support for these children and families is important to overcome this common problem. The school teachers also need to be educated on this matter. In the West a nurse would visit the school and educate the staff. This improves the support and the care they receive at the school. In future we would be able to get the help of diabetes educator nurses to take part in educating not only families of diabetic patients but also the school teachers.

\section{Exercise and sick day management}

Hypoglycaemia is commonly seen during or after exercise. Help of a trained dietician and frequent monitoring of blood glucose are important aspects in the management. It is important to check blood glucose more frequently during sick days and to check ketone bodies when blood glucose is more than $15 \mathrm{mmol} / \mathrm{l}$. We do not have facilities to check blood ketone levels with the available glucometers. Parents are advised to keep a constant contact with the diabetes educator nurses or medical professionals during this time. Non availability of these resources increases the risk of admissions with DKA during an illness. However, we do not have the necessary data to assess whether this is true in Sri Lanka

\section{Psychological support}

The diagnosis of diabetes in a child is a very distressing event for the entire family. The feeling of guilt, denial, blaming each other within the family should be addressed at the very beginning. The psychological well being of the child is important to avoid anxiety, conduct disorders, eating disorders, and depression which could affect the long term diabetes control. Constant assessment by a paediatric psychologist during follow up will help to identify these problems early.

The various myths related to the management of these children in Sri Lanka also need to be addressed. Some families believe that indigenous medicine and or religious activities would cure diabetes. At times it has been very challenging to overcome these deep rooted myths and beliefs. It should be emphasised to the parents that management of diabetes in children is different to that of adults. As childhood diabetes was not common in our society in the past adults with diabetes have become their role models which could at times be dangerous. The diabetes educator nurse will be of great help in this situation.

\section{Support groups}

Diabetes support groups could organise constant educational programmes and camps for young patients. It will also provide a platform to share the experience, worries about diabetes with peers. These support groups offer an invaluable service for the patients and their parents in Western countries. Time has come for us to initiate support groups for chronic conditions like diabetes in Sri Lanka.

\section{How could we overcome the challenges in the management of T1DM in children?}

1. There is a need to identify the incidence and disease burden of T1DM in Sri Lanka.

2. Make newer insulin products available at a reasonable price or provide these from the state hospitals.

3. Have better insulin delivery devices, needles and blood glucose monitoring facilities available for children.

4. Improve the facilities for screening of complications of diabetes.

5. Develop multidisciplinary dedicated clinics.

6. Have a trained nurse available for 24 hours to help patients and families. These nurses need to be available in all parts of the country.

7. Paediatric dieticians who are trained on carbohydrate counting, management of hypoglycaemia during exercise should be made available at least in the main teaching hospitals.

8. Ongoing educational programmes with the use of media, seminars, diabetic camps for young children and their families.

9. Child friendly informational leaflets, videos to help them understand what is happening in their body, why they need insulin.

10. Practical sessions on carbohydrate counting or selecting the appropriate portion diet for the individual patient

11. Educate school teachers.

12. Make available the services of a psychiatrist on a regular basis even if the child or the family members do not show signs of psychological problems.

13. Have separate clinics for adolescents with diabetes to cater for their specific problems.

14. Public awareness programmes regarding occurrence and treatment of T1DM to overcome deep rooted myths.

15. Have national guidelines for investigation of a child with diabetes at presentation, management of T1DM, management of diabetic ketoacidosis, subsequent care and follow up screening of a child with diabetes, management of hypoglycaemia, management of sick days and exercise.

16. Continuing medical education of health care professionals. 
17. Initiate support groups for patients and parents.

Improperly managed diabetes in children is a silent debilitating condition which would reduce the life expectancy and increase the morbidity making them a burden to the country. Time has come for us as health care professionals to speak up on behalf of our future generation to make a difference.

\section{References}

1. Patterson CC, Dahlquist GG, Gyurus E, Green A, Soltesz G. Incidence trends for childhood type 1 diabetes in Europe during 1989-2003 and predicted new cases 2005-20: a multicentre prospective registration study. Lancet 2009: 373: $2027-33$.

2. Sabbah E, Savola K, Ebeling T, et al. Genetic, autoimmune, and clinical characteristics of childhood and adult-onset type 1 diabetes. Diabetes Care 2000: 23: 1326 - 32.

3. Barrett JC, Clayton DG, Concannon P, et al. Genome wide association study and meta-analysis find that over 40 loci affect risk of type1 diabetes. Nat Genet 2009.

4. Bao F, Yu L, Babu S, et al. One third of HLA DQ2 homozygous patients with type 1 diabetes express celiac disease associated transglutaminase autoantibodies. $J$ Autoimmun 1999; 13: 143 -8.

5. Myhre AG, Undlien DE, Lovas K, et al. Autoimmune adrenocortical failure in Norway: autoantibodies and human leukocyte antigen class II associations related to clinical features. J Clin Endocrinol Metab 2002; 87: 618- 23.
6. Sagen JV, Raeder H, Hathout E, et al. Permanent neonatal diabetes due to mutations in KCNJ11 encoding Kir 6.2: patient characteristics and initial response to sulfonylurea therapy. Diabetes 2004; 53: 2713 - 8.

7. Njolstad PR, Sovik O, Cuesta-Munoz A, et al. Neonatal diabetes mellitus due to complete glucokinase deficiency. $N$ Engl J Med 2001; 344: 1588-92.

8. Stoffers DA, Zinkin NT, Stanojevic V, Clarke WL, Habener JF. Pancreatic agenesis attributable to a single nucleotide deletion in the human IPF1 gene coding sequence. Nat Genet 1997; 15: 106 - 10.

9. Wildin RS, Ramsdell F, Peake J, et al. X-linked neonatal diabetes mellitus, enteropathy and endocrinopathy syndrome is the human equivalent of mouse scurfy. Nat Genet 2001; 27: 18-20.

10. Delepine M, Nicolino M, Barrett T, et al. EIF2AK3, encoding translation initiation factor 2-alpha kinase 3, is mutated in patients with Wolcott-Rallison syndrome. Nat Genet 2000; 25: 406 -9.

11. The Diabetes Control and Complications Trial Research Group. The effect of intensive treatment of diabetes on the development and progression of long term complications in insulin-dependent diabetes mellitus. New England Journal of Medicine 1993: 329: 977-86.

12. White NH, Cleray PA, Dahms W, Goldstein D, Malone J, Tamborlane WV. Beneficial effects of intensive therapy of diabetes during adolescence: outcomes after the conclusion of the Diabetes Control and Complications Trial (DCCT). J Pediatr 2001; 139: 804 - 12. 